\title{
Chronic Contusion Spinal Cord Injury Impairs Ejaculatory Reflexes in Male Rats: Partial Recovery by Systemic Infusions of Dopamine D3 Receptor Agonist 7OHDPAT
}

\author{
Natalie Kozyrev, ${ }^{1,2}$ Michael D. Staudt, Arthur Brown,,3 and Lique M. Coolen ${ }^{1,2,4,5}$
}

\begin{abstract}
Chronic spinal cord injury (SCI) causes major disruption of ejaculatory function in men. Ejaculation is a reflex and the spinal generator for ejaculatory reflexes in the rat has been located in the lumbosacral spinal cord. The effects of SCI on the rat spinal ejaculation generator and ejaculatory reflexes remain understudied. The first goal of the current study was to establish the effects of chronic SCI on the function of the spinal ejaculation generator. Male rats received a contusion injury of the spinal cord at spinal level T6-T7. Ejaculatory reflexes elicited by electrical stimulation of the dorsal penile nerve (DPN) were evaluated in injured and control rats at 4-6 weeks following SCI. SCI males demonstrated significant reductions in bursting of the bulbocavernosus muscle (BCM), an indicator for expulsion phase of ejaculation, and in seminal vesicle pressure (SVP) increases, an indicator for the emission phase of ejaculation, following DPN stimulation. Thus, contusion SCI resulted in long-term impairment of ejaculatory reflexes.The D3 agonist 7-hydroxy-2-(di- $N$-propylamino) tetralin (7OHDPAT) facilitates ejaculation in spinal cord intact rats, thus the second goal of the current study was to test whether subcutaneous infusions of 7OHDPAT can facilitate ejaculatory reflexes in rats with chronic SCI. Male rats received a contusion injury at T6-T7 and effects of systemic administration of 7OHDPAT $(1 \mathrm{mg} / \mathrm{kg})$ were tested $4-5$ weeks following injury. Results showed that 7OHDPAT administration facilitated ejaculatory reflexes in SCI males with or without DPN stimulation, provided that supraspinal inputs to the lumbar cord were severed by transection just prior to evaluating the reflex. Thus, 7OHDPAT administration in SCI males was able to overcome the detrimental effects of SCI on ejaculatory reflexes.
\end{abstract}

Key words: neuroplasticity; sensory function; spinal cord injury; traumatic spinal cord injury

\section{Introduction}

C HRONIC SPINAL CORD INJURY (SCI) causes major disruption of ejaculatory function in men. ${ }^{1}$ A small portion of men with SCI $(9 \%)$ can ejaculate by masturbation, ${ }^{2}$ whereas the vast majority of men with SCI require medical procedures, that is, penile vibratory stimulation or electroejaculation, to induce ejaculation. ${ }^{3,4} \mathrm{Re}-$ covery of normal sexual function is the highest priority for paraplegic men, followed by bladder function. ${ }^{5-7}$ However, the neural mechanisms that alter ejaculatory function following chronic SCI are currently unknown. ${ }^{4}$

Ejaculation is a complex physiological process that is composed of two successive phases: emission and expulsion. ${ }^{8}$ During the emission phase, seminal fluids are secreted from the accessory sexual organs, including the prostate, seminal vesicles, and vas deferens, and moved through the urethra, while the bladder neck and external urethral sphincter shut to deter retrograde ejaculation. During the expulsion phase, the striated perineal muscles, including the bulbocavernosus muscle (BCM), undergo coordinated, rhythmic contractions and in this way vigorously eject seminal contents from the urethral meatus. ${ }^{9-12}$

Ejaculation is a reflex ${ }^{13,14}$ controlled by a spinal ejaculation generator. $^{8,15-23}$ In male rats, the spinal ejaculation generator comprises a set of interneurons located in lamina 10 and the medial aspect of lamina 7 in lumbar segments 3 and 4 (L3-L4) that project to spinal autonomic and motor neurons. ${ }^{8,15-23}$ These interneurons are known as lumbar spinothalamic (LSt) cells due to their anatomical positioning in the lumbar spinal cord and thalamic

\footnotetext{
${ }^{1}$ Department of Anatomy and Cell Biology, Western University, London, Ontario, Canada.

${ }^{2}$ Department of Physiology, University of Michigan, Ann Arbor, Michigan.

${ }^{3}$ Robarts Research Institute, Western University, London, Ontario, Canada.

${ }^{4}$ Department of Neurobiology and Anatomical Sciences, ${ }^{5}$ Department of Physiology and Biophysics, University of Mississippi Medical Center, Jackson, Mississippi.
} 
projections, ${ }^{16,24,25}$ and they are critical for ejaculation. Previous studies from our laboratory have shown that targeted lesions of these interneurons completely ablate ejaculation while sparing all other components of male sexual behavior in freely moving male rats during coitus. ${ }^{15}$ Furthermore, lesioning LSt cells also abolishes ejaculatory reflexes induced by stimulation of sensory inputs from the dorsal penile nerve (DPN) or urethra, or triggered by infusion of the selective dopamine D3 receptor agonist 7-hydroxy-2-(di- $\mathrm{N}$ propylamino) tetralin (7OHDPAT) in anesthetized and spinalized male rats. ${ }^{21}$ The mechanisms whereby the spinal ejaculation generator controls ejaculation involve the coordination of sympathetic, parasympathetic, and motor ouflow necessary to trigger emission and expulsion ${ }^{17,26-28}$ as well as the integration of the DPNtransmitted sensory signals during mating with this autonomic and motor ouflow to initiate ejaculation. ${ }^{8,17,27}$ Specifically, the LSt cells are postulated to convert sensory signals associated with mating into motor or secretory outputs. ${ }^{15,17,21,27}$ LSt cell axons directly project to sympathetic, parasympathetic, and motor spinal nuclei in the thoracolumbar and lumbosacral spinal cord implicated in emission and expulsion components of ejaculation. ${ }^{18,22,23,29-31}$

Despite the need to further investigate the effects of chronic SCI on ejaculatory function and the recent advances in our understanding of the spinal ejaculation generator, the effects of SCI on the spinal ejaculation generator remain understudied. Therefore, the first goal of the current study was to establish the effects of chronic SCI on the function of the spinal ejaculation generator in a rat model of contusion SCI. The contusion injury model uses a single brief displacement of the spinal cord and has been demonstrated to closely replicate the key pathophysiological features of human injury by producing either prolonged, or rapidly applied, extradural compression. ${ }^{32-34}$ This is a clinically relevant paradigm, as human SCI is rarely caused by complete transection, and is often due to crush injuries resulting from compression of the spinal cord. ${ }^{35}$ The function of the spinal ejaculation generator is best studied utilizing physiological paradigms to study parameters of emission and expulsion. ${ }^{8,36}$ The paradigm utilized in the current study triggers ejaculatory reflexes by electrical stimulation of the DPN. ${ }^{13,37-39}$ In all mammals, including rats, ${ }^{13}$ apes, ${ }^{40}$ and humans, ${ }^{41}$ ejaculation can be triggered by DPN stimulation. ${ }^{41}$ Electromyography (EMG) recordings of the $\mathrm{BCM}$ demonstrate bursting patterns identical to those observed during the expulsion phase of naturally occurring ejaculation, ${ }^{10}$ whereas increased pressure in the seminal vesicles is highly correlated with BCM bursting and considered an accurate measure of the emission phase. ${ }^{36}$ Indeed, in anesthetized, spinalized rats, DPN stimulation elicits rhythmic contractions of the BCM similar to those in a behaving animal, ${ }^{19-23}$ and reflexive penile erections are evident. ${ }^{13,42}$ Moreover, removal of supraspinal inputs is required to trigger the reflex, ${ }^{36}$ therefore providing a unique opportunity to investigate the complex mechanisms of the control of ejaculation by the spinal generator.

The neurotransmitter dopamine has also been suggested to play a critical role in ejaculation. ${ }^{43}$ It has been demonstrated that intracerebroventricular (i.c.v.) or systemic infusions of the selective dopamine D3 receptor agonist 7OHDPAT reduces the number of intromissions prior to ejaculation as well as the latency to ejaculation in freely mating male rats. ${ }^{44,45}$ In addition, i.c.v. administration of 7OHDPAT consistently triggers coordinated, rhythmic contractions of the BCM and concurrent increases in seminal vesicle pressure (SVP) in anesthetized spinal intact male rats. $^{21,43,46-48}$ 7OHDPAT can also trigger ejaculatory reflexes in spinalized anesthetized male rats, demonstrating that it acts on D3 receptors in the lumbosacral spinal cord to mediate ejaculation. ${ }^{21}$
Moreover, effects of 7OHDPAT are dependent on intact LSt cells, suggesting that this D3 agonist acts either on LSt cells or on D3 receptor-containing neurons that provide inputs to LSt cells. ${ }^{21}$

Given the facilitatory effects of 7OHDPAT on ejaculatory reflexes, the second objective of the current study aimed to test whether subcutaneous infusions of the D3 receptor agonist 7OHDPAT can facilitate ejaculatory reflexes in male rats with chronic SCI. It was predicted that subcutaneous infusion of 7OHDPAT would restore ejaculatory reflexes in chronic SCI male rats without added sensory stimulation by acting on D3 receptors in the lumbosacral spinal cord. Additionally, it was predicted that subcutaneous infusions of 7OHDPAT would rescue ejaculatory reflexes in SCI males following DPN stimulation.

\section{Methods}

\section{Animals}

Adult male Sprague Dawley rats (225-250g) were obtained from Charles River Laboratories (Sherbrooke, Quebec, Canada or Wilmington, MA) and pair housed in standard housing cages on a 12-h light/dark cycle with lights off at 11 a.m. Food and water were available at all times. All procedures were approved by the Animal Care Committee at the University of Western Ontario and the Use and Care of Animals Committee at the University of Michigan and conformed to the guidelines outlined by the the Canadian Council on Animal Care and the United States National Institutes of Health.

\section{Spinal cord surgeries}

Male rats were divided in SCI or sham groups. All surgical procedures were performed under ketamine $(87 \mathrm{mg} / \mathrm{mL}) / x y l a z i n e$ $(13 \mathrm{mg} / \mathrm{mL})$ anesthesia $(1 \mathrm{~mL} / \mathrm{kg}$ i.p. injection). The back of the males was shaved and cleaned with betadine, iodine, and $70 \%$ alcohol solution. Eyes were covered with Lacri-lube ${ }^{\circledR}$ opthalamic ointment (Allergen, Irvine, CA) to prevent them from drying. All animals received a laminectomy between the sixth and seventh thoracic spinal segments (T6-T7). SCI male rats received a contusion injury of the spinal cord, performed with the IH-400 impactor (Precision Systems and Instrumentation, LLC, Lexington, $\mathrm{KY}$ ). The impactor was positioned 1.5 inches above the cord with the tip at approximately $5 \mathrm{~mm}$ above the cord, and a force of 200 Kdyn was applied to the dura of the exposed spinal cord. The cord displacement values ranged from 1287 to $1763 \mu \mathrm{m}$ (mean $=1610.5$; standard error of mean $[\mathrm{SEM}]=67.82)$. The velocity $(\mathrm{mm} / \mathrm{sec})$ values ranged from 117 to 153 (mean $=130.44, \mathrm{SEM}=6.22)$. The actual force values ( $\mathrm{K}$ dynes) ranged from 200 to 212 (mean= 204.89; SEM =1.35). Dwell time was 0 sec. Afterwards, the layers of muscle and skin were sutured separately, with absorbable sutures and wound clips respectively. To alleviate pain and prevent bacterial infection, animals received subcutaneous injections subcutaneously (s.c.) of the analgesic ketoprofen $(5 \mathrm{mg} / \mathrm{mL} / \mathrm{kg})$, and the antibiotic Baytril ${ }^{\circledR}(5 \mathrm{mg} / \mathrm{mL} / \mathrm{kg})$, immediately following surgery and for 3 days thereafter. The sham animals underwent all procedures, including laminectomy, and ketoprofen and Baytril treatment, except for the contusion injury. All SCI animals had bladder dysfunction and bladders were manually emptied by light compression twice per day until bladder function returned (typically 710 days after injury). Sham animals were handled, but bladders were not expressed.

\section{Locomotor testing}

In all experiments, all SCI animals had notable partial paralysis of hind limbs. To quantify this motor impairment, in experiment 1 , motor function was measured in an open field test for hind limb function using the Basso, Beattie, and Bresnahan (BBB) scale. ${ }^{49}$ $\mathrm{BBB}$ scores were recorded 6 times during the first 4 weeks 
following contusion injury. Groups were compared with a repeated measures analysis of variance (ANOVA). In experiment 2, locomotor activity was measured at 3 and 4 weeks after contusion SCI or sham procedures, in an Open Field Apparatus (Med Associates Inc., St. Albans City, Vermont) surrounded by two $16 \times 16$ photobeam arrays, during the light phase for a 15-min duration. Locomotor activity was measured and expressed as ambulatory distance in the horizontal plane, and vertical rearing (in time, seconds). Both measures of locomotoractivity were analyzed using a two-way repeated ANOVA (factors: SCI and time) and Holm-Sidak post hoc tests using $95 \%$ confidence levels.

\section{Recording parameters of expulsion and emission}

Male rats were deeply anesthetized with urethane $(1.5 \mathrm{~g} / \mathrm{kg}$, i.p.) and BCM activity and SVP were recorded as described previously. ${ }^{19,22,23}$ Connective tissues and ligaments enclosing the reproductive organs were carefully removed to expose the muscle of the BCM and the DPN. In preparation for EMG recordings, silver recording electrodes connected to the PowerLab/7SP Data Acquisition System (AD Instruments, Inc., Colorado Springs, CO) were inserted bilaterally into the $\mathrm{BCM}$ and a ground electrode was implanted into the muscle of the right thigh. For simultaneous recordings of SVP, a marker of the emission component of ejaculation, ${ }^{22,23,43}$ a small abdominal incision was performed and the right seminal vesicle was located. SVP was recorded using a pressure catheter (AD Instruments Inc., model number: SPR-671, 1.4 F, Single, Straight, $15 \mathrm{~cm}, \mathrm{Ny}$ ) coupled to a catheter interface cable (AD Instruments, Inc., model number: AEC-IOD) and connected to a Bridge AMP (AD Instruments, Inc.). The pressure catheter was gently inserted into the lumen of the seminal vesicle and fixed in place before the start of recordings. The initial electrical stimulation of the DPN comprised square wave pulses of $1 \mathrm{msec}$ duration, $4 \mathrm{~V}$ at 30 or $60 \mathrm{~Hz}$ for $10 \mathrm{sec}$. These established DPN stimulation parameters consistently induce emission and expulsion reflexes in all salinetreated control male rats, reflected in rhythmic increases in SVP and concurrent bursting of the BCM, respectively. ${ }^{19-23}$

\section{BCM EMG and SVP analysis}

SVP and BCM EMG recordings were analyzed for $15 \mathrm{~min}$ following infusions of saline and 7OHDPAT, and for $90 \mathrm{sec}$ following each DPN stimulation. Specifically, the numbers of BCM events and bursts and concurrent SVP increases were analyzed following DPN stimulation and subcutaneous infusion of saline or 7OHDPAT. The numbers of BCM events were analyzed using LabChart 7.35 (AD Instruments, Inc.) software and all events above baseline activity (i.e., spikes above baseline activity) were incorporated in the analysis. ${ }^{19,22,23}$ The numbers of BCM bursts and SVP increases were analyzed as previously described. ${ }^{22,23}$ Specifically, eight or more successive BCM events above baseline activity (and without retrun to baseline between events) were counted as a "burst" and all increases in SVP that were greater than baseline and that occurred simultaneously with BCM bursts were incorporated in the analysis. These parameters were determined in our previous publications to accurately represent ejaculatory reflexes. ${ }^{19,21-23}$ Finally, we have previously determined that repeated DPN stimulations and intrathecal infusions do not disrupt the ability to trigger ejaculatory reflexes and thus within animal comparisons are a powerful method of analysis. $^{22,23}$

\section{Acute spinal transection}

Depending on the experimental design (see below), animals received a complete spinal transection acutely before or after 7OHDPAT infusions. A laminectomy was performed at the eighth thoracic segment (T8), caudal to the contusion injury site, and the spinal cord was completely transected. The purpose of this acute transection was to study the spinal ejaculatory generator in isolation from supraspinal inhibitory influences. ${ }^{19-23,36}$

\section{Drug infusions}

7OHDPAT (Sigma Alderich Co. LLC) was dissolved in sterile saline $(0.9 \%)$ and injected s.c. at a dose of $1 \mathrm{mg} / \mathrm{mL} / \mathrm{kg}$. This dose was previously shown to trigger ejaculatory reflexes in anesthetized, spinalized male rats. ${ }^{21}$

\section{Experimental design}

Experiment 1: Effects of SCI on DPN-stimulated ejaculatory reflexes. The main objective of this first experiment was to determine the effects of chronic contusion injury on ejaculatory reflexes triggered by DPN stimulation. First, either 4 or 6 weeks after SCI or sham treatment, anesthetized SCI males with contusion injuries ( $n=6 ; 4$ weeks and $n=6 ; 6$ weeks), sham males ( $n=4 ; 4$ and 6 weeks), and surgery-intact control males ( $n=4 ; 4$ and 6 weeks) received an acute spinal tranection at T8 to remove supraspinal influence on the ejaculation generator. Two $h$ later, all males received DPN stimulation at 30 and $60 \mathrm{~Hz}$ (parameters that reliably trigger ejaculation in control animals). ${ }^{19,22,23}$ In addition, all males received superthreshold stimulations of 120 and $180 \mathrm{~Hz}$ to test if higher stimulation frequencies were required in SCI males (Fig. 1A). Each animal received the four stimulations of $30 / 60$ and $120 / 180 \mathrm{~Hz}$ with 5-min intervals between stimulations. The order of the four stimulations varied for each animal, to counter balance the order. BCM EMG activity was recorded and analysed for $90 \mathrm{sec}$ following each stimulation. SVP was not recorded in this first experiment.

Statistical analysis. Numbers of BCM events and bursts were compared between the three groups (sham, SCI 4 weeks, SCI 6 weeks) within each of the stimulation parameters using ANOVA on ranks and Dunn's method for pairwise comparisons with the sham control group.

Experiment 2: 7OHDPAT or saline infusions after acute spinal transection. The main goal of this experiment was to test the facilitative effects of 7OHDPAT on ejaculatory reflexes in SCI males. First, 4-5 weeks after SCI or sham treatment, anesthetized SCI males with contusion injuries $(n=6)$ and sham males $(n=6)$ received an acute spinal tranection at $\mathrm{T} 8$ to remove supraspinal influence on the ejaculation generator. Two h later, all males received DPN stimulation at 30 and $60 \mathrm{~Hz}$ in Trial 1 (Fig. 2A), in a counterbalanced manner with 5-min intervals between stimulations and SVP and BCM EMG were recorded for $90 \mathrm{sec}$ following each stimulation. The goal was to determine effects of SCI on the ability of DPN stimulation to trigger ejaculatory reflexes.

Next, sterile saline $(1 \mathrm{~mL} / \mathrm{kg})$ was infused s.c. and SVP and BCM EMG were recorded for $15 \mathrm{~min}$. Subsequently, 7OHDPAT $(1 \mathrm{mg} / \mathrm{mL} / \mathrm{kg})$ was infused s.c. and once again SVP and BCM EMG were recorded for $15 \mathrm{~min}$ following drug infusion (Fig. 3A). The goal of this part of the experiment was to determine the effects of SCI on 7OHDPAT-induced ejaculatory reflexes. No effects of saline on ejaculatory reflexes were expected, and thus it served as the vehicle control for the 7OHDPAT infusions.

Next, $15 \mathrm{~min}$ following 7OHDPAT infusion, all animals received 30 and $60 \mathrm{~Hz}$ DPN stimulations in Trial 2 in a counterbalanced order and BCM EMG and SVP were recorded for $90 \mathrm{sec}$ following each DPN stimulation (Fig. 2A). The goal of this second DPN stimulation trial was to test if 7OHDPAT influenced the ability of DPN stimulation to trigger ejaculatory reflexes in SCI males (compared with Trial 1).

Statistical analysis. First, the effects of 30 and $60 \mathrm{~Hz}$ DPN stimulation on numbers of BCM events and bursts, and SVP 


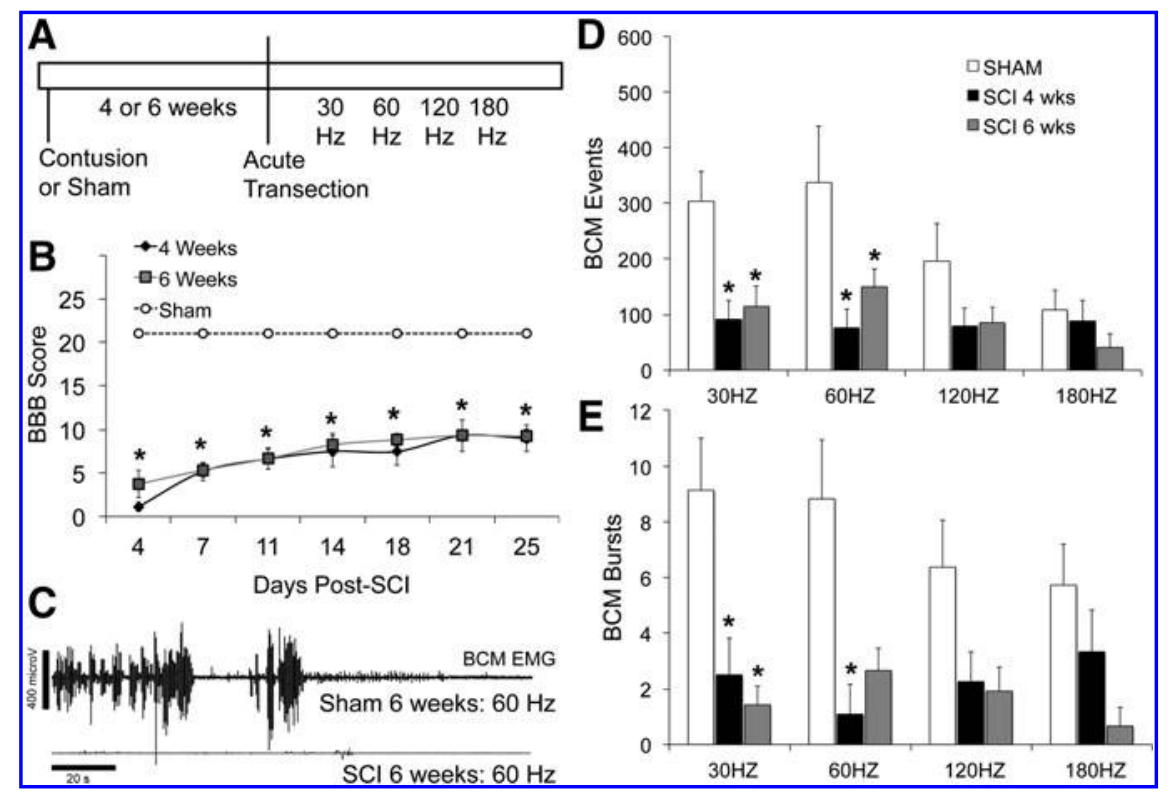

FIG. 1. Effects of contusion injury on ejaculatory reflex triggered by DPN stimulation. Time line and experimental design of experiment 1 are shown in (A). Animals received contusion injury or sham surgery and were tested for BBB scores during a period of 4 weeks. Four or six weeks after contusion or sham injury, all males received a spinal transection acutely prior to testing ejaculatory reflexes triggered by four stimulations of the DPN of four different frequencies, each separated by $5 \mathrm{~min}$ and delivered in varying order. BBB scores are shown in (B) at seven times following contusion or sham surgeries of males tested for ejaculatory reflexes at $4(n=6)$ or 6 weeks $(n=6)$ following contusion injury (SCI). Sham males did not differ from surgery intact males and were combined into one sham group $(n=8 ; 4$ sham and 4 intact). * indicates significant different for both SCI groups from sham $(p<0.05)$. (C) Representative BCM EMG traces $(90$ sec $)$ of sham and SCI males tested at 6 weeks following surgery and following $60 \mathrm{~Hz}$ stimulation of the DPN, illustrating the effects of SCI on the bursting patterns of the BCM. Quantitative analysis of BCM events (D) and bursts (E) triggered by 30, 60, 120, and 180 Hz DPN stimulations. * denotes significant differences from sham group within each DPN stimulation frequency. BBB, Basso, Beattie, and Bresnahan; BCM, bulbocavernosus muscle; DPN, dorsal penile nerve; EMG, electromyography; SCI, spinal cord injury.

increases in sham and SCI males in Trial 1 were analyzed using a two-way ANOVA (factors: SCI vs. sham; 30 versus $60 \mathrm{~Hz}$ stimulations; Fig. 2A Trial 1). Second, the effects of 7OHDPAT infusions in SCI and sham animals on numbers of BCM events and bursts and SVP increases in the absence of DPN stimulations were tested using a two-way repeated measures ANOVA (factors: SCI vs. sham; 7OHDPAT vs. saline; Fig. 3A). Third, the effects of SCI and 7OHDPAT on responses to DPN stimulation were compared using two-way ANOVAS separately for 30 and for $60 \mathrm{~Hz}$ stimulation parameters (factors: SCI vs. sham, and 7OHDPAT (Trial 2) vs. no drug (Trial 1); Fig. 2A). Holms Sidak tests were used for all post hoc analyses. For all tests, a 95\% confidence level was set.

Experiment 3: 7OHDPAT infusions before and after acute spinal transection. In the third experiment, the effects of 7OHDPAT were tested in SCI males prior to an acute spinal transection (Trial 1) as well as after an acute spinal transection (Trial 2; Fig. 4A). First, sham $(n=7)$ and SCI $(n=8)$ male rats animals received infusion s.c. of sterile saline $(1 \mathrm{~mL} / \mathrm{kg})$ and SVP and BCM EMG were recorded for $15 \mathrm{~min}$ after saline infusion (Trial 1: saline). Subsequently, all rats received injections s.c. of 7OHDPAT $(1 \mathrm{mg} / \mathrm{mL} / \mathrm{kg})$ and SVP and BCM EMG were recorded for 15 min immediately following drug infusion (Trial 1: 7OHDPAT). After a rest period of $3 \mathrm{~h}$, all animals received an acute and complete transection at T8 (acute transection). Two $\mathrm{h}$ after the transection, all males received sterile saline and SVP and BCM EMG were recorded for 15 min following saline injection (Trial 2: saline). Subsequently, 7OHDPAT $(1 \mathrm{mg} / \mathrm{mL} / \mathrm{kg})$ was infused s.c. and SVP and BCM EMG were recorded for another $15 \mathrm{~min}$ following drug infusion (Trial 2: 7OHDPAT).

Finally, $15 \mathrm{~min}$ following 7OHDPAT infusion, all animals received 30 and $60 \mathrm{~Hz}$ DPN stimulations in a counterbalanced order and BCM EMG and SVP were recorded for 90 sec following each DPN stimulation. The goal of this last manipulation was to repeat and confirm the findings from experiment 2 .

Statistical analysis. First, the effects of 7OHDPAT (vs. saline) in sham and SCI animals were determined for numbers of SVP increases, BCM events, and bursts using a two-way repeated measures ANOVA (factors: SCI vs. sham; 7OHDPAT vs. saline; Fig. 4). Effects were analyzed separately for data collected before the acute spinal transection (Trial 1) and after spinal transection (Trial 2). Second, the effects of the acute transection (Trial 1 vs. Trial 2) were analyzed using a two-way repeated ANOVA to compare the differences in BCM events and bursts and SVP increases induced by 7OHDPAT before and after the transection (factors: SCI vs. sham; before transection (Trial 1) vs. after transection (Trial 2; Fig. 4A). Finally, the effects of 30 and $60 \mathrm{~Hz}$ DPN stimulations were compared for each of the ejaculation parameters between Sham and SCI males using $t$ tests, for 30 and $60 \mathrm{~Hz}$ separately.

\section{Results}

\section{Chronic contusion injury impaired locomotor activity}

Locomotor activity was impaired following contusion injury, but not sham injury. This was evident by reduced BBB scores 4 and 6 weeks after contusion injury in experiment 1 (Fig. 1B) and significantly lower levels of ambulatory activity and impaired ability to rear and thus time spent on vertical activity, compared with sham animals at 3 and 4 weeks after contusion injury in experiment 2 (Table 1 ). 
Table 1. Locomotor Activity Impairments in SCI Male Rats

\begin{tabular}{lcc}
\hline & Horizontal distance $(\mathrm{cm})$ & Vertical time $(\mathrm{sec})$ \\
\hline Sham 3 weeks & $100.65 \pm 9.75$ & $165.68 \pm 30.16$ \\
SCI 3 weeks & $64.57 \pm 10.64^{\mathrm{a}}$ & $111.98 \pm 35.19$ \\
Sham 4 weeks & $109.79 \pm 18.13$ & $195.56 \pm 31.96$ \\
SCI 4 weeks & $68.12 \pm 10.68^{\mathrm{a}}$ & $107.82 \pm 26.05^{\mathrm{a}}$ \\
\hline
\end{tabular}

Locomotor activity 3 and 4 weeks after contusion SCI or sham procedures in experiment 2, expressed as ambulatory distance in the horizontal plane (Horizontal distance in centimeters) and vertical rearingin time (Vertical time in seconds).

${ }^{a}$ Indicates significant difference from sham controls at the same time point. SCI, spinal cord injury.

\section{Chronic contusion injury impaired ejaculatory reflexes}

In the first experiment, ejaculatory reflexes were tested at 4 or 6 weeks following SCI. SCI caused significant impairments in BCM events and bursts following 30 and $60 \mathrm{~Hz}$ stimulations of the DPN and numbers of BCM events and bursts were significantly lower compared with sham controls (Fig. $1 \mathrm{C}-\mathrm{E}$; $\mathrm{H}=7.2-10.7$; $p=0.03-0.005)$. Higher DPN stimulation frequencies of 120 and $180 \mathrm{~Hz}$ were not sufficient to overcome the impairment and induce ejaculatory reflexes in SCI males (Fig. 1 D,E). At these higher DPN stimulation frequencies, SCI males did not significantly differ from sham animals, but this was due to an inability of the higher DPN stimulation frequencies to trigger robust ejaculatory reflexes in sham males. These data indicate that chronic contusion injury at mid-lower thoracic levels causes severe impairments in the expulsion phase of the ejaculatory reflex.

\section{OHDPAT infusions improved ejaculatory reflexes in chronic SCI males upon DPN stimulation}

In the second experiment, the findings from experiment 1 were confirmed and further expanded by demonstrating that SCI also impaired the emission phase of ejaculatory reflexes, that is, increases in SVP. DPN stimulation at 30 or $60 \mathrm{~Hz}$ (in Trial 1; Fig. 2A) did not trigger ejaculatory reflexes in SCI males (Fig. 2B,C; Trial 1). There were main effects of SCI on BCM events $(\mathrm{F}(1,20)=110.03$; $p<0.001$; Fig. 2F), BCM bursts $(\mathrm{F}(1,20)=112.23 ; p<0.001$; Fig. $2 \mathrm{G})$, and SVP increases $(\mathrm{F}(1,20)=55.36 ; p<0.001$; Fig. $2 \mathrm{H})$. Both 30 and $60 \mathrm{~Hz}$ DPN stimulations resulted in significantly lower

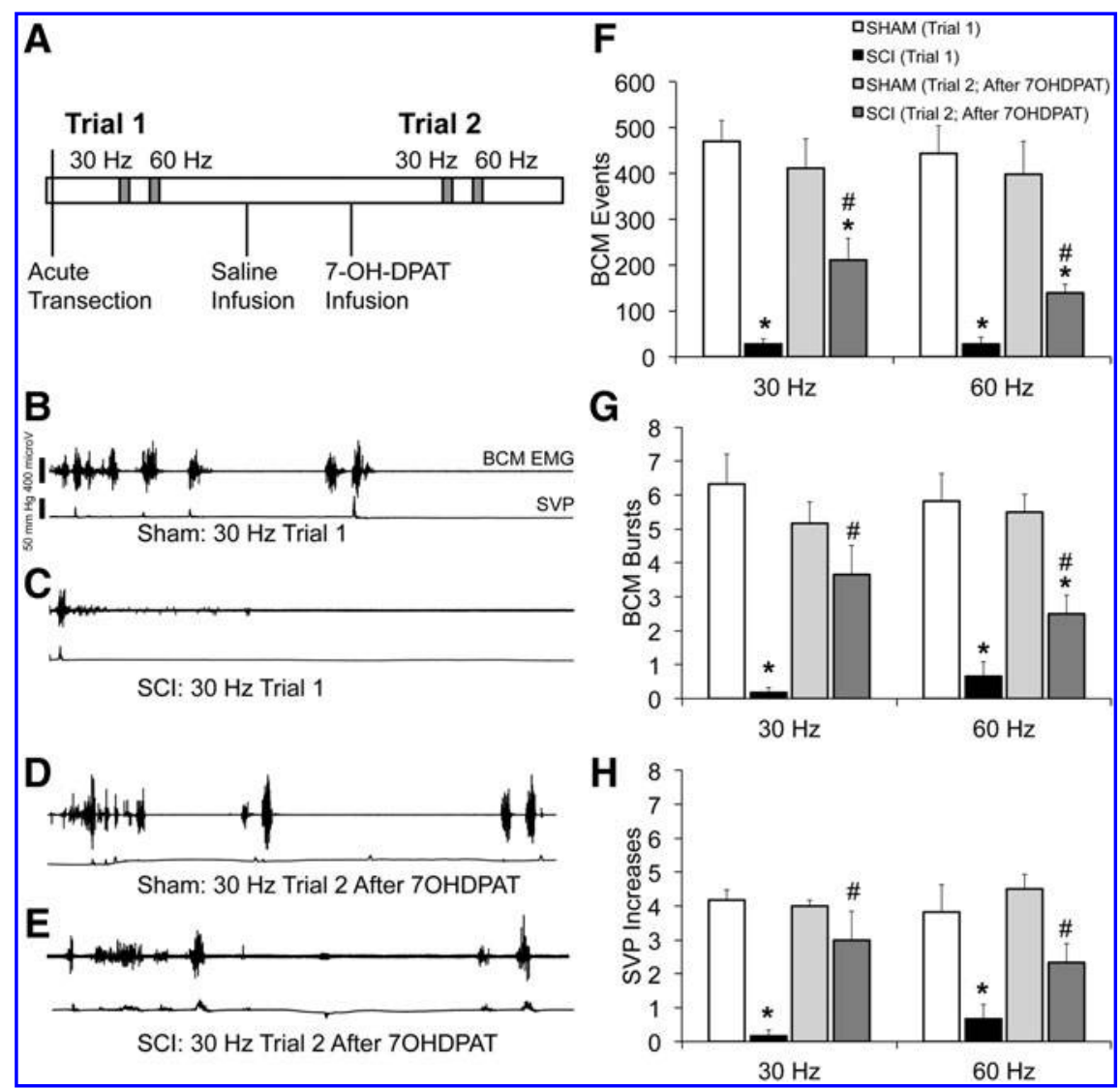

FIG. 2. Effects of contusion injury on expulsion and emission parameters triggered by DPN stimulation. Time line and experimental design of experiment 2 are shown in (A). Four to five weeks after sham or contusion injury, all males recived an acute spinal tranestion, followed by two DPN stimulations (30-60 Hz; in varying order) to trigger ejaculatory reflexes. Subsequently, males received infusions of saline followed by 7 OHDPAT (results shown in Fig. 3), again followed by two DPN stimulations (30-60 Hz; in varying order) to trigger ejaculatory reflexes. Representative BCM EMG (top) and SVP (bottom) traces $(90 \mathrm{sec})$ in response to $30 \mathrm{~Hz}$ DPN stimulation in Trial 1 (B,C) and following 7OHDPAT infusion in Trial 2 (D,E) in a sham male (B-D; same animal) and a contusion injury (SCI) male (C-E; same animal). Quantitative analysis of BCM events (F) bursts $(\mathbf{G})$ and SVP $(\mathbf{H})$ triggered by 30 and $60 \mathrm{~Hz}$ DPN stimulations in Trial 1 and following infusions of $1 \mathrm{mg} / \mathrm{kg}$ 7OHDPAT (Trial 2). * denotes significant differences between sham and SCI within the same testing trial and DPN stimulation frequency. \# indicates significant differences from trial within the same treatment group and DPN stimulation. 7OHDPAT, 7-hydroxy-2-(di- $N$-propylamino) tetralin; BCM, bulbocavernosus muscle; DPN, dorsal penile nerve; EMG, electromyography; SCI, spinal cord injury; SVP, seminal vesicle pressure. 


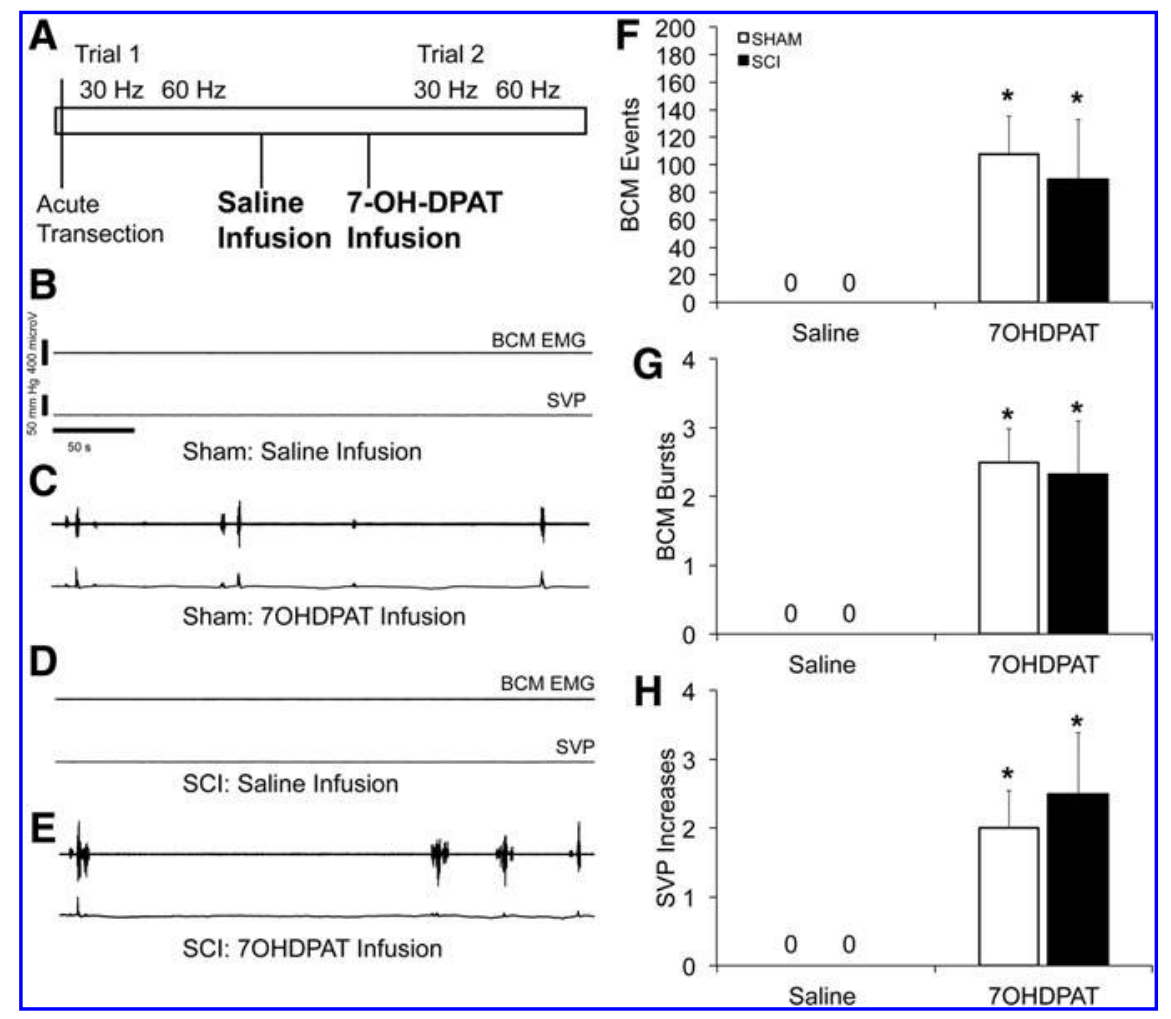

FIG. 3. Effects of 7OHDPAT infusions on ejaculatory reflex in sham and SCI male rats. Time line and experimental design of experiment 2 are again shown in (A), highlighting the timing of saline and 7OHDAT infusions. Representative BCM EMG (top) and SVP (bottom) traces in response to saline (B-D) and 7OHDPAT (C-E) infusion are shown in a sham male (B,C; same animal) and a contusion injury (SCI) male (D,E; same animal). Traces are shown for a 300-sec period after the occurrence of the first event after 7OHDPAT infusion or the equivalent time after saline. Quantitative analysis of BCM events $(\mathbf{F})$, bursts $(\mathbf{G})$, and SVP $(\mathbf{H})$ in sham and SCI male rats triggered by infusions of $1 \mathrm{mg} / \mathrm{kg}$ 7OHDPAT. Because saline infusions did not cause any events, bursts, or SVP increases, zeros are placed in the absence of bars. * indicates significant differences from saline within the sham or SCI treatment group. 7OHDPAT, 7-hydroxy-2-(di- $N$ propylamino) tetralin; BCM, bulbocavernosus muscle; EMG, electromyography; SCI, spinal cord injury; SVP, seminal vesicle pressure.

numbers of BCM events, bursts, and SVP increases in SCI males compared with sham $(p<0.001)$, demonstrating detrimental effects of SCI on ejaculatory reflexes triggered by DPN stimulation.

However, 7OHDPAT amplified ejaculatory reflexes following 30 and $60 \mathrm{~Hz}$ DPN stimulation in SCI males (in Trial 2; Fig. 2A). Whereas DPN stimulation in Trial 1 (without drug infusion) failed to trigger any BCM activity or SVP increases in SCI males, DPN stimulation following 7OHDPAT infusions triggered ejaculatory reflexes in SCI males comparable to those in sham males (Fig. 2D,E). Statistical analysis using two-way repeated ANOVA (factors SCI and Trial) showed main effects of SCI (vs. sham) on the numbers of BCM events (Fig. 2F), bursts (Fig. 2G), and SVP increases (Fig. 2H), following both DPN stimulation frequencies $(30 \mathrm{~Hz}: \mathrm{F}(1,10)=46.677$; $p<0.001 ; \mathrm{F}(1,10)=23.1 ; p<0.001 ; \mathrm{F}(1,10)=46.677 ; p<0.001$, respectively; $60 \mathrm{~Hz}: \mathrm{F}(1,10)=29.091 ; p<0.001 ; \mathrm{F}(1,10)=47.45$; $p<0.001 ; \mathrm{F}(1,10)=29.09 ; p<0.001$, respectively). Moreover, there were significant interactions between factors of 7OHDPAT treatment (i.e., Trial 1 vs. Trial 2) and SCI (vs. sham) on the numbers of SVP increases, BCM events, and bursts following $30 \mathrm{~Hz}$ DPN stimulations (Fig. 2F-H; $\mathrm{F}(1,10)=5.9 ; p=0.036 ; \mathrm{F}(1,10)=12.484 ; p=0.005$; $\mathrm{F}(1,10)=7.17 ; p=0.023$, respectively).

Post hoc analyses showed that following 7OHDPAT infusions, SCI males had significantly greater numbers of BCM events, bursts, and SVP increases, following $30 \mathrm{~Hz}$ DPN stimulation (Trial 2) compared with the same stimulation but without 7OHDPAT in Trial 1 (Fig. 2F-H; $p=0.014-0.004$ ). In addition, BCM bursts and
SVP increases in SCI males did not significantly differ from sham males in Trial 2, showing that SCI males fully responded to 7OHDPAT. However, BCM events in SCI males were significantly lower compared with sham males in Trial 2 (BCM events: $p=0.002$ ). 7OHDPAT treatment did not alter responses to $30 \mathrm{~Hz}$ DPN stimulation in sham animals as ejaculatory reflexes in sham animals did not differ between Trails 1 and 2 (Fig. 2F-H), thus effects of 7OHDPAT were specific to SCI males.

Increases in BCM activity and SVP were also noted following $60 \mathrm{~Hz}$ DPN stimulation after 7OHDPAT treatment (Trial 2, Fig. $2 \mathrm{~F}-\mathrm{H})$, with a significant increase compared with Trial 1 without drug, for BCM bursts $(p=0.031)$, SVP increases $(p=0.005)$, and a trend for events $(p=0.055)$. However, in Trial 2 after 7OHDPAT, BCM activity and SVP increases in SCI males were significantly lower compared with sham males (SVP: $p=0.020$; Events: $p=0.002$; Bursts: $p=0.001$ ). Together, these findings demonstrate that DPN stimulation following 7OHDPAT treatment was effective in triggering ejaculatory reflexes in SCI males.

\section{OHDPAT infusions improved ejaculatory reflexes in chronic SCI males in the absence of sensory stimulation}

Following the first DPN stimulation Trial (Trial 1; Fig. 3A), all animals received infusions of saline followed by infusions of 7OHDPAT to determine the effects of the D3 receptor agonist on BCM activity and SVP increases (Fig. 3A). Saline infusions did not 


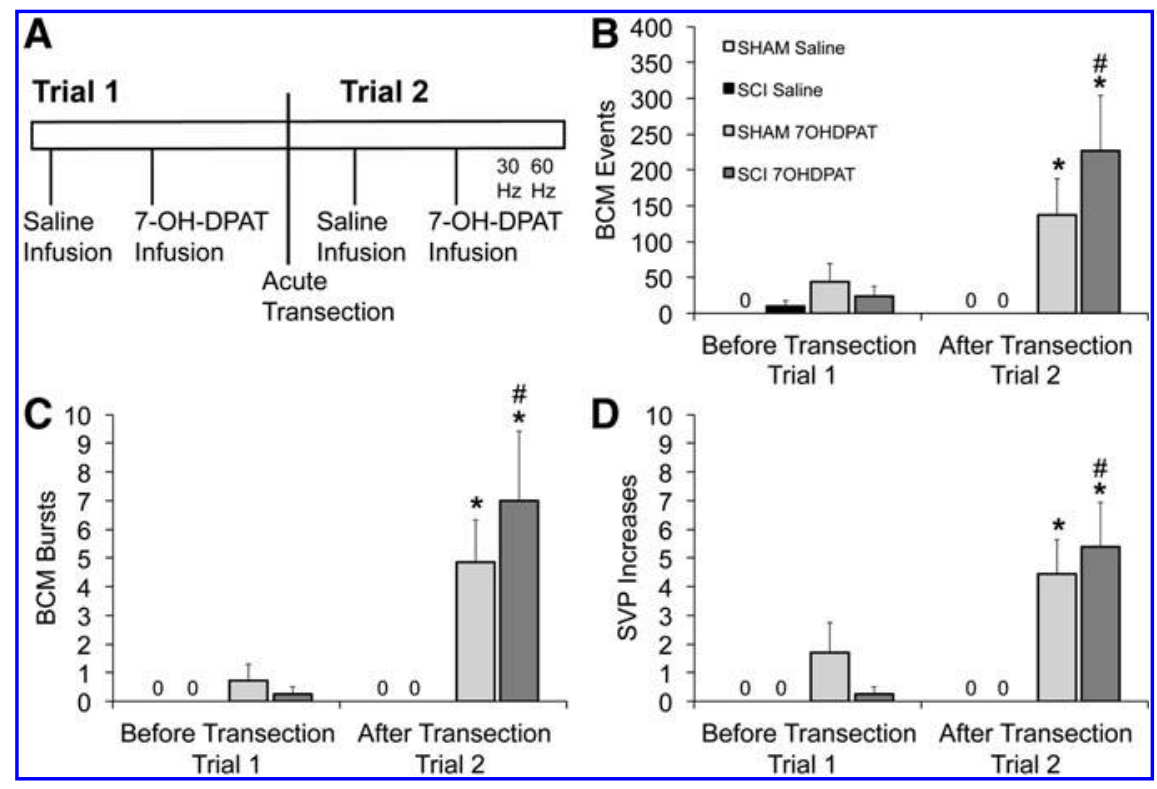

FIG. 4. Effects of acute spinal transection on ejaculatory reflex triggered by 7-OH-DPAT infusions in sham and SCI male rats. Time line and experimental design of experiment 3 are shown in (A). Four to five weeks following contusion or sham injury, all males received infusions of saline followed by 7OHDPAT. Next, all males recived an acute spinal transection, again followed by infusions of saline and 7OHDPAT. Finally, all males received two DPN stimulations $(30-60 \mathrm{~Hz}$; in varying order) to trigger ejaculatory reflexes (results shown in Table 2). Quantitative analysis of BCM events (B), bursts (C), and SVP (D) in sham and contusion injury (SCI) male rats triggered by infusions of saline or 7OHDPAT. Where saline infusions did not cause any events, bursts, or SVP increases, zeros are placed in the absence of bars. * indicates significant differences in Trial 2 from saline within the sham or SCI treatment group. \# denotes significant differences within the 7OHDPAT-treated SCI group between Trials 1 and 2. 7OHDPAT, 7-hydroxy-2-(di- $N$-propylamino) tetralin; BCM, bulbocavernosus muscle; SCI, spinal cord injury; SVP, seminal vesicle pressure.

cause any increases in BCM or SVP in sham or SCI males as expected (Fig. 3B,D). In contrast, 7OHDPAT infusions triggered ejaculatory reflexes in sham male rats (Fig. 3C). Moreover, the effects of 7OHDPAT in SCI males were similar to the effects in sham males (Fig. 3E). In particular, qualitative analysis of BCM events and bursts and SVP increases showed main effects of 7OHDPAT treatment (Fig. 3F-H; $\mathrm{F}(1,20)=14.85 ; p<0.001 ; \mathrm{F}(1.20)=17.163$; $p<0.001 ; \mathrm{F}(1,20)=12.484 ; p=0.002$, respectively). Post hoc analyses showed that both sham and SCI males had significantly greater numbers of BCM events (Fig. 3F), BCM bursts (Fig. 3G), and SVP increases (Fig. 3H) following infusions of 7OHDPAT compared with saline infusions (Events: sham: $p=0.007$; SCI: $p=0.022$; Bursts: sham: $p=0.007$; SCI: $p=0.010$; SVP increases: sham: $P=0.031$; SCI: $p=0.014$ ). Finally, the latencies to onset of BCM events after 7OHDPAT infusions did not differ between sham and SCI males (126.4 $\pm 21 \mathrm{sec}$ in sham and $118.3 \pm 27.4 \mathrm{sec}$ in SCI males).

These results indicate that sham and SCI groups did not differ in their responses to 7OHDPAT, and thus that the D3 agonist triggered ejaculatory reflexes in SCI males similar to effects in sham males. Of all sham males tested, $83 \%$ responded to 7OHDPAT infusion alone, compared with $100 \%$ of SCI males. "Responders" had at least one SVP increase and accompanying BCM burst following 7OHDPAT treatment.

\section{OHDPAT infusion effects before and after acute transection}

The first goal of the third experiment was to test the effects of 7OHDPAT infusions in SCI males without an acute transection (Trial 1; Fig. 4A). A two-way ANOVA analysis on effects of 7OHDPAT (vs. saline) in SCI (vs. sham) males prior to the spinal transection (Trial 1) revealed statistical trends of 7OHDPAT infusion on the numbers of BCM events and SVP increases (Fig. 4B,D; $p=0.054$ and 0.061 , respectively), but failed to reach significance. Thus, 7OHDPAT infusions were not effectively triggering ejaculatory reflexes in sham or SCI males without removal of supraspinal influences on the spinal ejaculation generator.

However, there were significant effects of 7OHDPAT following the acute spinal transection (Trial 2; Fig. 4A), on BCM events (Fig. 4B) and bursts (Fig. 4C), and SVP increases (Fig. 4D; F(1, 26)= 14.498; $p<0.001 ; \mathrm{F}(1,26)=16.136 ; p<0.001 ; \mathrm{F}(1,26)=23.607$; $p<0.001$, respectively). Post hoc tests showed that 7OHDPAT increased BCM events and bursts and SVP increases compared with saline in SCI males $(p=0.002-<0.001)$ and sham males ( $p=0.033-0.006)$. There were no significant differences between sham and SCI males in any of the measures of ejaculatory reflexes.

Because 7OHDPAT appeared to be less effective prior to transection than after the acute transection, a two-way repeated ANOVA was used to directly compare the effects of SCI (vs. sham) and the two trials (before transection vs. after transection) on the ability of 7OHDPAT to induce ejaculatory reflexes. Indeed, there was an effect of acute transection (Trial 1 vs. 2) on BCM events and bursts and SVP increases (Fig. 4B-D; $\mathrm{F}(1,13)=7.86 ; p=0.015$; $\mathrm{F}(1,13)=12.04 ; p=0.004 ; \mathrm{F}(1,13)=9.49 ; p=0.009$, respectively $)$. There were no main effects of SCI. Pairwise comparisons revealed that SCI males responded with greater numbers of BCM events (Fig. 4B), bursts (Fig, 4C), and SVP increases (Fig. 4D) after the acute transection (Trial 2) compared with before the acute transection (Trial 1) ( $p=0.015,0.008,0.011$, respectively). In contrast, there were no significant differences between the two trials in sham males. These results suggest that SCI males require an acute spinal transection for optimal effects of 7OHDPAT infusions. 
TABle 2. EfFEcts of DPN Stimulation After 7OHDPAT

\begin{tabular}{lccc}
\hline & BCM events & BCM bursts & SVP increases \\
\hline Sham $30 \mathrm{~Hz}$ & $193.7 \pm 75.5$ & $3.3 \pm 0.3$ & $3.0 \pm 0.6$ \\
SCI $30 \mathrm{~Hz}$ & $195.8 \pm 53.2$ & $4.8 \pm 1.2$ & $4.25 \pm 0.8$ \\
Sham $60 \mathrm{~Hz}$ & $194.5 \pm 40.6$ & $3.2 \pm 0.6$ & $2.8 \pm 0.3$ \\
SCI $60 \mathrm{~Hz}$ & $327.1 \pm 59.7$ & $5.1 \pm 0.8$ & $4.3 \pm 0.7$ \\
\hline
\end{tabular}

Quantitative analysis of BCM events, BCM bursts, and SVP increases in sham and contusion injury (SCI) male rats triggered by either $30 \mathrm{~Hz}$ or $60 \mathrm{~Hz}$ DPN stimulations after infusion of 7OHDPAT, in experiment 3 . No significant differences were detected between sham and SCI males.

BCM, bulbocavernosus muscle; DPN, dorsal penile nerve; SCI, spinal cord injury; SVP, seminal vesicle pressure.

Finally, following 7OHDPAT infusions, all sham and SCI males received 30 and $60 \mathrm{~Hz}$ DPN stimulations to further replicate and confirm the findings from experiment 2 that 7OHDPAT facilitates the ability of DPN stimulation to trigger ejaculatory reflexes in SCI males. Indeed, DPN stimulations triggered similar ejaculatory reflexes in both sham and SCI males and no significant differences were found between sham and SCI males (Table 2).

\section{Discussion}

These studies demonstrate that chronic contusion SCI at midthoracic levels in male rats caused severe impairment of ejaculatory reflexes triggered by DPN stimulation. Moreover, systemic administration of the dopamine D3 receptor agonist 7OHDPAT improved ejaculatory reflexes in SCI male rats. Specifically, infusions of 7OHDPAT facilitated ejaculatory reflexes in SCI males induced by DPN stimulation. In addition, 7OHDPAT infusions triggered emission and expulsion reflexes in SCI males in the absence of sensory stimulation, provided an acute spinal transection was applied to remove supraspinal influences. Thus, 7OHDPAT administration in SCI males was able to overcome some of the detrimental effects of SCI on ejaculatory reflexes.

The current study demonstrates that contusion injury at midthoracic level results in long-term deficits of ejaculatory function in male rats. Previous studies in anesthetized rats have shown effects of select spinal lesions or lateral hemisections on erectile reflexes and pudendal nerve reflexes. ${ }^{50-52}$ The current study advances and extends the current knowledge of SCI effects on ejaculatory function by providing a detailed characterization of effects of chronic SCI on parameters of the ejaculatory reflex and by using an injury paradigm that closely resembles human SCI. ${ }^{32-34}$ Therefore, the current results suggest that chronic contusion injury causes disruptions at the level of the spinal ejaculation generator. The spinal ejaculation generator consists of LSt cells with axonal projections to autonomic and motor neurons involved in emission and expulsion. LSt cell bodies are located at spinal levels L3-L4 $4^{8,15,17,27}$ and LSt axons are located in proximity to preganglionic autonomic cells in the interomedial lateral cell column, dorsal central nucleus, and sacral parasympathetic nucleus. ${ }^{16,22}$ LSt cells and axons co-contain the neuropeptides enkephalin, galanin, cholecystokinin, and gastrin releasing peptide (GRP). ${ }^{16,22,24,25,29,31,53}$ Release of these neuropeptides from their axons is critical for ejaculation, as blockade of the neuropeptide receptors for GRP or enkephalin in the lumbosacral spinal cord prevents ejaculatory reflexes triggered by DPN stimulation. ${ }^{22,23} \mathrm{SCI}$ or lesions in humans at or below the L3 level resulted in the most severe impairment of ejaculatory function, providing support for the role of the spinal ejaculation generator in humans. ${ }^{4}$ In the current study, direct injury to the spinal ejaculation generator is unlikely as contusion injury was conducted at the mid-thoracic levels. However, damage to descending supraspinal pathways may have resulted in long-term plasticity in the spinal ejaculation generator at the lower spinal levels.

The spinal control center is under descending inhibitory and excitatory influence of supraspinal sites. ${ }^{47,54}$ These sites include the medial preoptic area, ${ }^{55,56}$ paraventricular nucleus of the hypothalamus, ${ }^{57} \mathrm{nu}-$ cleus paragigantocellularis, ${ }^{58,59}$ and the periaqueductal gray. ${ }^{60} \mathrm{SCI}$ at thoracic levels likely causes damage to these descending pathways. ${ }^{52,61}$ However, because the current study used a physiological model in which the reflex generator was isolated from supraspinal influences at time of analysis, the ejaculatory deficits observed are not a direct effect of damage to these descending pathways, but may rather reflect long-term changes in the spinal generator influenced by changes in descending inputs. ${ }^{62}$ Impaired descending pathways can lead to the denervation of spinal neurons promoting the release of neurotrophic factors leading to synaptic remodeling and plasticity of afferent neurons, marked changes in the properties of these neurons, and their connection. ${ }^{63}$ Hence, it is probable that damage to the descending pathways controlling the spinal ejaculation generator caused long-term plasticity, a hypothesis to be tested in future studies.

The spinal ejaculation generator receives sensory inputs, and the current findings that DPN stimulation in SCI rats did not result in ejaculatory reflexes suggests disrupted transmission of sensory information from the DPN to the spinal ejaculation generator. The precise nature of the afferent stimuli that activate the spinal ejaculation generator and thus trigger ejaculation is unknown and may involve somatosensory, visceral sensory, proprioceptive, temperature-related, and/or noxious inputs. ${ }^{64}$ The majority of the spinal afferents involved in mediating ejaculation travel via the DPN, a sensory branch of the pudendal nerve. ${ }^{17,27,65,66}$ Stimulation of the DPN produces coordinated and rhythmic contractions of the BCM akin to those observed in male rats during mating ${ }^{13}$ and bilateral lesion of the DPN completely ablates ejaculation in copulating rats. ${ }^{67}$ Moreover, stimulation of the DPN results in the neural activation of LSt cells, in anesthetized and spinalized male rats. ${ }^{19,22,23}$ Anatomically, it is known that fibers of the DPN innervate the dorsal horn and the dorsal central gray in lumbosacral spinal cord, in the vicinity of the LSt cells. ${ }^{68,69}$ However, the precise mechanisms by which sensory information associated with mating or DPN stimulation is transmitted to the LSt cells are presently unknown. The neurotransmitter glutamate appears to be critical for the relay of sensory inputs to LSt cells as ejaculation or DPN stimulation activates NMDA receptors expressed by LSt cells. ${ }^{20}$ Moreover, pharmacological blockade of NMDA receptors completely prevents ejaculatory reflexes triggered by DPN stimulation in male rats, whereas intrathecal infusions of $N$-methyl-D-aspartate (NMDA) stimulate ejaculatory reflexes. $^{20}$

The current findings suggest that SCI at mid-thoracic levels caused disrupted transmission of sensory informationto the spinal ejaculation generator, potentially caused by a reorganization of the sensory fibers in the DPN that relay sensory information from the sexual organs to the dorsal horn of the lumbosacral spinal cord. Indeed, previous studies reported reorganization of sensory fibers of the micturition reflex pathway in cats following chronic $\mathrm{SCI}^{63,70-72}$ indicating that reorganization of afferent fibers may also occur in other reflex pathways in the spinal cord, namely in the spinal ejaculation generator. The DPN is composed of $\mathrm{A} \delta$ and Cfibers ${ }^{73}$ but the role of each fiber type in ejaculation is presently unknown. However, previous studies reported changes in soma size, neuropeptide expression, and function of C-fiber afferents, ${ }^{74}$ as well as neuroplasticity of sensory fibers in the lumbosacral spinal 
cord in rats with chronic SCI. ${ }^{75,76}$ Therefore, we hypothesize that the inefficacy of DPN stimulation to trigger ejaculatory reflexes in chronic SCI males may be the result of synaptic remodeling of Cfibers in the DPN.

A main finding of the current study is that systemic infusions of the D3 receptor agonist 7OHDPAT in SCI males can stimulate ejaculatory reflexes in the absence of DPN stimulation, or facilitate the effects of DPN stimulation to trigger ejaculatory reflexes as long as the supraspinal inputs have been removed. The sites of action of 7OHDPAT are not clearly understood. 7OHDPAT can trigger coordinated BCM bursting following i.c.v. infusions ${ }^{46}$ or targeted injections into the medial preoptic area. ${ }^{77}$ However, previous studies from our laboratory have shown that subcutaneous infusions of 7OHDPAT in anesthetized and spinally transected male rats triggered ejaculatory reflexes, ${ }^{21}$ providing evidence for actions of 7OHDPAT within the spinal ejaculation generator. Moreover, LSt lesions blocked ejaculatory reflexes induced by 7OHDPAT suggesting that the D3 receptor agonist acts at the level of LSt cells and/or on neurons in the spinal cord that convey sensory signals associated with mating or DPN stimulation to the LSt cells. $^{21}$ This observation is in agreement with a hypothesis that chronic SCI causes alterations and impairments of relay of sensory inputs to LSt cells and that these can be at least partially overcome by stimulation of D3 receptors involved in this sensory processing. Future studies will be needed to determine the exact localization of D3 receptors. Finally, even though 7OHDPAT alleviated some of the effects of SCI on ejaculatory reflexes, this effect was dependent on removal of the supraspinal inputs by use of an acute spinal transection at T8. It is possible that higher dosages of $\mathrm{D} 3$ receptor agonist are required to trigger ejaculatory reflexes in SCI males, especially because sham animals also did not respond to 7OHDPAT in the current study. The current studies were conducted with a single dosage of 7OHDPAT, previously shown to be effective in control, non-surgery males, to reliably trigger ejaculatory reflexes, but tested following an acute spinal transection to isolate the spinal reflex generator. $^{21}$

In conclusion, the current study demonstrated that contusion injury causes severe deficits in ejaculatory function, despite full or partial recovery of bladder function and locomotion. Moreover, it was shown that systemic administration of the dopamine D3 receptor agonist 7OHDPAT restored ejaculatory reflexes in anesthetized and spinalized male rats with chronic SCI. The findings lead to several testable hypotheses regarding effects of contusion injury on reorganization and plasticity of the DPN following chronic spinal trauma and disruption of transmission of sensory information via the DPN to the spinal ejaculation generator. Finally, the current data suggest that $\mathrm{D} 3$ receptor activation may be a potential avenue for development of treatment of ejaculatory dysfunction in men with chronic SCI.

\section{Author Disclosure Statement}

No competing financial interests exist.

\section{References}

1. Brackett, N.L., Ferrell, S.M., Aballa, T.C., Amador, M.J., Padron, O.F., Sonksen, J., and Lynne, C.M. (1998). An analysis of 653 trials of penile vibratory stimulation in men with spinal cord injury. $\underline{\mathrm{J} \text {. Urol. }}$ 159, 1931-1934.

2. Kathiresan, A.S.Q., Ibrahim, E., Modh, R., Aballa, T.C., Lynne, C.M., and Brackett, N.L. (2012). Semen quality in ejaculates produced by masturbation in men with spinal cord injury. Spinal Cord 50, 891-894.
3. Brackett, N.L., Ibrahim, E., Iremashvili, V., Aballa, T.C., and Lynne, C.M. (2010). Treatment for ejaculatory dysfunction in men with spinal cord injury: an 18-year single center experience. J. Urol. 183, 23042308.

4. Chéhensse, C., Bahrami, S., Denys, P., Clément, P., Bernabé, J., and Giuliano, F. (2013). The spinal control of ejaculation revisited: a systematic review and meta-analysis of anejaculation in spinal cord injured patients. Hum. Reprod. Update 19, 507-526.

5. Anderson, K.D. (2004). Targeting recovery: priorities of the spinal cord-injured population. J. Neurotrauma 21, 1371-1383.

6. Anderson, K.D., Borisoff, J.F., Johnson, R.D., Stiens, S.A., and Elliott, S.L. (2007). The impact of spinal cord injury on sexual function: concerns of the general population. Spinal Cord 45, 328-337.

7. Anderson, K.D., Borisoff, J.F., Johnson, R.D., Stiens, S.A., and Elliott, S.L. (2007). Long-term effects of spinal cord injury on sexual function in men: implications for neuroplasticity. Spinal Cord 45, $338-348$.

8. Veening, J.G., and Coolen, L.M. (2014). Neural mechanisms of sexual behavior in the male rat: emphasis on ejaculation-related circuits. Pharmacol. Biochem. Behav. 121, 170-183.

9. Bohlen, J.G., Held, J.P., and Sanderson, M.O. (1980). The male orgasm: pelvic contractions measured by anal probe. Arch. Sex Behav. $9,503-521$

10. Gerstenberg, T.C., Levin, R.J., and Wagner, G. (1990). Erection and ejaculation in man. Assessment of the electromyographic activity of the bulbocavernosus and ischiocavernosus muscles. Br. J. Urol. 65, 395-402.

11. Holmes, G.M., Chapple, W.D., Leipheimer, R.E., and Sachs, B.D. (1991). Electromyographic analysis of male rat perineal muscles during copulation and reflexive erections. Physiol. Behav. 49, 12351246.

12. Holmes, G.M., and Sachs, B.D. (1991). The ejaculatory reflex in copulating rats: normal bulbospongiosus activity without apparent urethral stimulation. Neurosci. Lett. 125, 195-197.

13. Pescatori, E.S., Calabro, A., Artibani, W., Pagano, F., Triban, C., and Italiano, G. (1993). Electrical stimulation of the dorsal nerve of the penis evokes reflex tonic erections of the penile body and reflex ejaculatory responses in the spinal rat. J. Urol. 149, 627-632.

14. McKenna, K.E. (1999). Ejaculation. Encycl. Reprod., 1002-1008.

15. Truitt, W.A., and Coolen, L.M. (2002). Identification of a potential ejaculation generator in the spinal cord. Science (80-.) 297, 15661569.

16. Truitt, W.A., Shipley, M.T., Veening, J.G., and Coolen, L.M. (2003). Activation of a subset of lumbar spinothalamic neurons after copulatory behavior in male but not female rats. J. Neurosci. 23, 325331.

17. Coolen, L.M., Allard, J., Truitt, W.A., and McKenna, K.E. (2004). Central regulation of ejaculation. Physiol. Behav. 83, 203-215.

18. Allard, J., Truitt, W.A., McKenna, K.E., and Coolen, L.M. (2005). Spinal cord control of ejaculation. World J. Urol. 23, 119-126.

19. Staudt, M.D., de Oliveira, C. V, Lehman, M.N., McKenna, K.E., and Coolen, L.M. (2010). Activation of MAP kinase in lumbar spinothalamic cells is required for ejaculation. J. Sex. Med. 7, 24452457.

20. Staudt, M.D., de Oliveira, C. V, Lehman, M.N., McKenna, K.E., and Coolen, L.M. (2011). Activation of NMDA receptors in lumbar spinothalamic cells is required for ejaculation. J. Sex. Med. 8, 10151026.

21. Staudt, M.D., Truitt, W.A., McKenna, K.E., de Oliveira, C.V., Lehman, M.N., Coolen, L.M. (2012). A pivotal role of lumbar spinothalamic cells in the regulation of ejaculation via intraspinal connections. J. Sex. Med. 9, 2256-2265.

22. Kozyrev, N., Lehman, M.N., and Coolen, L.M. (2012). Activation of gastrin-releasing peptide receptors in the lumbosacral spinal cord is required for ejaculation in male rats. J. Sex. Med. 9, 13031318.

23. Kozyrev, N., Coolen, L.M. (2015). Activation of mu or delta opioid receptors in the lumbosacral spinal cord is essential for ejaculatory reflexes in male rats. PLoS One 10,1-18.

24. Ju, G., Melander, T., Ceccatelli, S., Hökfelt, T., and Frey, P. (1987). Immunohistochemical evidence for a spinothalamic pathway cocontaining cholecystokinin- and galanin-like immunoreactivities in the rat. Neuroscience 20, 439-456.

25. Coolen, L.M., Veening, J.G., Wells, A.B., and Shipley, M.T. (2003). Afferent connections of the parvocellular subparafascicular thalamic 
nucleus in the rat: evidence for functional subdivisions. J. Comp. Neurol. 463, 132-156.

26. Carro-Juarez, M., Cruz, S.L., and Rodriguez-Manzo, G. (2003). Evidence for the involvement of a spinal pattern generator in the control of the genital motor pattern of ejaculation. Brain Res. 975, 222-228.

27. Coolen, L.M. (2005). Neural control of ejaculation. J. Comp. Neurol. 493, 39-45

28. Carro-Juarez, M., and Rodriguez-Manzo, G. (2008). The spinal pattern generator for ejaculation. Brain Res. Rev. 58, 106-120.

29. Newton, B.W. (1993). Galanin immunoreactivity in rat spinal lamina IX: emphasis on sexually dimorphic regions. Peptides 14, 955-969.

30. Xu, C., Giuliano, F., Yaici, E.D., Conrath, M., Trassard, O., Benoit, G., and Verge, D. (2006). Identification of lumbar spinal neurons controlling simultaneously the prostate and the bulbospongiosus muscles in the rat. Neuroscience 138, 561-573.

31. Sakamoto, H., Matsuda, K., Zuloaga, D.G., Hongu, H., Wada, E., Wada, K., Jordan, C.L., Breedlove, S.M., and Kawata, M. (2008). Sexually dimorphic gastrin releasing peptide system in the spinal cord controls male reproductive functions. Nat. Neurosci. 11, 634-636.

32. Pearse, D.D., Lo, T.P., Cho, K.S., Lynch, M.P., Garg, M.S., Marcillo, A.E., Sanchez, A.R., Cruz, Y., and Dietrich, W.D. (2005). Histopathological and behavioral characterization of a novel cervical spinal cord displacement contusion injury in the rat. J. Neurotrauma 22, 680-702.

33. Gensel, J.C., Tovar, C.A., Hamers, F.P.T., Deibert, R.J., Beattie, M.S., and Bresnahan, J.C. (2006). Behavioral and histological characterization of unilateral cervical spinal cord contusion injury in rats. $\mathrm{J}$ Neurotrauma 23, 36-54.

34. Anderson, K.D., Sharp, K.G., and Steward, O. (2009). Bilateral cervical contusion spinal cord injury in rats. Exp. Neurol. 220, 9-22.

35. Bunge, M.B. (2008). Novel combination strategies to repair the injured mammalian spinal cord. J. Spinal Cord Med. 31, 262-269.

36. Giuliano, F., Pfaus, J., Srilatha, B., Hedlund, P., Hisasue, S., Marson, L., and Wallen, K. (2010). Experimental models for the study of female and male sexual function. J. Sex. Med. 7, 2970-2995.

37. Sachs, B.D., and Garinello, L.D. (1980). Hypothetical spinal pacemaker regulating penile reflexes in rats: evidence from transection of spinal cord and dorsal penile nerves. J. Comp. Physiol. Psychol. 94, 530-535.

38. Monaghan, E.P., and Breedlove, S.M. (1992). The role of the bulbocavernosus in penile reflex behavior in rats. Brain Res. 587, 178-180.

39. Yang, C.C., and Bradley, W.E. (1998). Peripheral distribution of the human dorsal nerve of the penis. J. Urol. 159, 1912-1917.

40. Herbert, J. (1973). The role of the dorsal nerves of the penis in the sexual behaviour of the male rhesus monkey. Physiol. Behav. 10, 293-300.

41. Wieder, J.A., Brackett, N.L., Lynne, C.M., Green, J.T., and Aballa, T.C. (2000). Anesthetic block of the dorsal penile nerve inhibits vibratory-induced ejaculation in men with spinal cord injuries. Urology 55, 915-917.

42. Rampin, O., Gougis, S., Giuliano, F., and Rousseau, J.P. (1997). Spinal Fos labeling and penile erection elicited by stimulation of dorsal nerve of the rat penis. Am. J. Physiol. 272, R1425-R1431.

43. Clement, P., Peeters, M., Bernabe, J., Denys, P., Alexandre, L., and Giuliano, F. (2008). Brain oxytocin receptors mediate ejaculation elicited by 7-hydroxy-2-(di-N-propylamino) tetralin (7-OH-DPAT) in anaesthetized rats. Br. J. Pharmacol. 154, 1150-1159.

44. Ahlenius, S., and Larsson, K. (1995). Effects of the dopamine D3 receptor ligand 7-OH-DPAT on male rat ejaculatory behavior. Pharmacol. Biochem. Behav. 51, 545-547.

45. Ferrari, F., and Giuliani, D. (1996). Behavioral effects induced by the dopamine D3 agonist 7-OH-DPAT in sexually-active and -inactive male rats. Neuropharmacology 35, 279-284.

46. Clément, P., Bernabé, J., Denys, P., Alexandre, L., and Giuliano, F. (2007). Ejaculation induced by i.c.v. injection of the preferential dopamine D3 receptor agonist 7-hydroxy-2-(di-N-propylamino) tetralin in anesthetized rats. Neuroscience 145, 605-610.

47. Giuliano, F., and Clement, P. (2005). Physiology of ejaculation: emphasis on serotonergic control. Eur. Urol. 48, 408-417.

48. Clement, P., Peeters, M., Bernabe, J., Laurin, M., Alexandre, L., and Giuliano, F. (2009). Role of the neurokinin-1 receptors in ejaculation in anesthetized rats. J. Sex. Med. 6, 126-134.

49. Basso, D.M., Beattie, M.S., and Bresnahan, J.C. (1995). A sensitive and reliable locomotor rating scale for open field testing in rats. $\underline{\mathbf{J}}$. Neurotrauma 12, 1-21.
50. Hubscher, C.H., and Johnson, R.D. (2006). Chronic spinal cord injury induced changes in the responses of thalamic neurons. Exp. Neurol. 197, 177-188.

51. Johnson, R.D. (2006). Descending pathways modulating the spinal circuitry for ejaculation: effects of chronic spinal cord injury. Prog. Brain Res. 152, 415-426.

52. Hubscher, C.H., and Johnson, R.D. (2000). Effects of acute and chronic midthoracic spinal cord injury on neural circuits for male sexual function. II. Descending pathways. J. Neurophysiol. 83, 25082518

53. Nicholas, A.P., Zhang, X., and Hökfelt, T. (1999). An immunohistochemical investigation of the opioid cell column in lamina $\mathrm{X}$ of the male rat lumbosacral spinal cord. Neurosci. Lett. 270, 9-12.

54. Marson, L., List, M.S., and McKenna, K.E. (1992). Lesions of the nucleus paragigantocellularis alter ex copula penile reflexes. Brain Res. 592, 187-192.

55. Hull, E.M., Du, J., Lorrain, D.S., and Matuszewich, L. (1997). Testosterone, preoptic dopamine, and copulation in male rats. Brain Res. Bull. 44, 327-333.

56. Pehek, E.A., Warner, R.K., Bazzett, T.J., Bitran, D., Band, L.C., Eaton, R.C., and Hull, E.M. (1988). Microinjection of cis-flupenthixol, a dopamine antagonist, into the medial preoptic area impairs sexual behavior of male rats. Brain Res. 443, 70-76.

57. Marson, L., and McKenna, K.E. (1994). Stimulation of the hypothalamus initiates the urethrogenital reflex in male rats. Brain Res. 638, 103-108.

58. Marson, L., and McKenna, K.E. (1992). A role for 5-hydroxytryptamine in descending inhibition of spinal sexual reflexes. Exp. Brain Res. 88, 313-320.

59. Normandin, J.J., and Murphy, A.Z. (2008). Nucleus paragigantocellularis afferents in male and female rats: organization, gonadal steroid receptor expression, and activation during sexual behavior. J. Comp. Neurol. 508, 771-794.

60. Rizvi, T.A., Murphy, A.Z., Ennis, M., Behbehani, M.M., and Shipley, M.T. (1996). Medial preoptic area afferents to periaqueductal gray medullo-output neurons: a combined Fos and tract tracing study. J. Neurosci. 16, 333-344.

61. Hubscher, C.H., and Johnson, R.D. (1999). Effects of acute and chronic midthoracic spinal cord injury on neural circuits for male sexual function. I. Ascending pathways. J. Neurophysiol. 82, 13811389

62. Petruska, J.C., Hubscher, C.H., and Rabchevsky, A.G. (2013). Challenges and opportunities of sensory plasticity after SCI. Front. Physiol. 4, 231.

63. De Groat, W.C., and Yoshimura, N. (2010). Changes in afferent activity after spinal cord injury. Neurourol. Urodyn. 29, 63-76.

64. Carro-Juarez, M., and Rodriguez-Manzo, G. (2005). Role of genital sensory information in the control of the functioning of the spinal generator for ejaculation. Int. J. Impot. Res. 17, 114-120.

65. Marberger, H. (1974). The mechanisms of ejaculation. Basic Life Sci. 4, Pt. B, 99-110

66. McKenna, K.E. (2001). Neural circuitry involved in sexual function. J. Spinal Cord Med. 24, 148-154.

67. Lodder, J., and Zeilmaker, G.H. (1976). Role of pelvic nerves in the postcopulatory abbreviation of behavioral estrus in female rats. J. Comp. Physiol. Psychol. 90, 925-929.

68. McKenna, K.E., and Nadelhaft, I. (1986). The organization of the pudendal nerve in the male and female rat. J. Comp. Neurol. 248, 532 549.

69. Tang, Y., Rampin, O., Giuliano, F., and Ugolini, G. (1999). Spinal and brain circuits to motoneurons of the bulbospongiosus muscle: retrograde transneuronal tracing with rabies virus. J. Comp. Neurol. 414, 167-192.

70. De Groat, W.C., Nadelhaft, I., Milne, R.J., Booth, A.M., Morgan, C., and Thor, K. (1981). Organization of the sacral parasympathetic reflex pathways to the urinary bladder and large intestine. J. Aut. Nerv. Syst. 3, 135-160.

71. De Groat, W.C., Kawatani, M., Hisamitsu, T., Cheng, C.L., Ma, C.P., Thor, K., Steers, W., and Roppolo, J.R. (1990). Mechanisms underlying the recovery of urinary bladder function following spinal cord injury. J. Auton. Nerv. Syst. 30 Suppl, S71-S77.

72. Araki, I., and de Groat, W.C. (1997). Developmental synaptic depression underlying reorganization of visceral reflex pathways in the spinal cord. J. Neurosci. 17, 8402-8407. 
73. Johnson, R.D., and Halata, Z. (1991). Topography and ultrastructure of sensory nerve endings in the glans penis of the rat. J. Comp. Neurol. 312, 299-310.

74. Yoshimura, N., Erdman, S.L., Snider, M.W., and De Groat, W.C. (1998). Effects of spinal cord injury on neurofilament immunoreactivity and capsaicin sensitivity in rat dorsal root ganglion neurons innervating the urinary bladder. Neuroscience $83,633-643$.

75. Zvarova, K., Dunleavy, J.D., and Vizzard, M.A. (2005). Changes in pituitary adenylate cyclase activating polypeptide expression in urinary bladder pathways after spinal cord injury. Exp. Neurol. 192, 46-59.

76. Vizzard, M.A. (2006). Neurochemical plasticity and the role of neurotrophic factors in bladder reflex pathways after spinal cord injury. Prog. Brain Res. 152, 97-115.

77. Kitrey, N.D., Clément, P., Bernabé, J., Alexandre, L., and Giuliano, F. (2007). Microinjection of the preferential dopamine receptor D3 agonist 7-hydroxy-N,N-di-n-propylaminotetralin hydrobromide into the hypothalamic medial preoptic area induced ejaculation in anesthetized rats. Neuroscience 149, 636-641.

Address correspondence to:

Lique M. Coolen, PhD

Departments of Physiology and Biophysics and Neurobiology and Anatomical Sciences University of Mississippi Medical Center 2500 North State Street

Jackson, MS 39216

E-mail:1coolen@umc.edu 


\section{This article has been cited by:}

1. Natalie Kozyrev, Lique M. Coolen. 2017. Activation of galanin and cholecystokinin receptors in the lumbosacral spinal cord is required for ejaculation in male rats. European Journal of Neuroscience 45:6, 846-858. [Crossref]

2. Marcalee Sipski Alexander, Lesley Marson. 2017. The neurologic control of arousal and orgasm with specific attention to spinal cord lesions: Integrating preclinical and clinical sciences. Autonomic Neuroscience . [Crossref]

3. Clément Chéhensse, Patricia Facchinetti, Stéphane Bahrami, Philippe Andrey, Jean-Marc Soler, Fabrice Chrétien, Jacques Bernabé, Pierre Clément, Pierre Denys, François Giuliano. 2017. Human spinal ejaculation generator. Annals of Neurology 81:1, 35-45. [Crossref]

4. Patrick Brice Deeh Defo, Elvis Asongu, Modeste Nya Wankeu, Esther Ngadjui, Georges Romeo Bonsou Fazin, François Xavier Kemka, Miguel Carro-Juarez, Albert Kamanyi, Pierre Kamtchouing, Pierre Watcho. 2017. Guibourtia tessmannii -induced fictive ejaculation in spinal male rat: involvement of D 1 , D 2 -like receptors. Pharmaceutical Biology 55:1, 1138-1143. [Crossref]

5. C. Chéhensse, P. Clément, C. Joussain, J. Bernabé, F. Giuliano. 2016. The spinal generator of ejaculation: Functional consequences of chronic spinalization and effect of substance P in anesthetized rats. Neuroscience 336, 12-19. [Crossref]

6. Ulf Simonsen, Simon Comerma-Steffensen, Karl-Erik Andersson. 2016. Modulation of Dopaminergic Pathways to Treat Erectile Dysfunction. Basic \& Clinical Pharmacology \& Toxicology . [Crossref] 